\title{
Aplicabilidade do termo antocrono para representar a velocidade de abertura de flores em inflorescência
}

\author{
Natalia Teixeira Schwab(1), Nereu Augusto Streck(1), Josana Andreia Langner ${ }^{(1)}$, \\ Bruna San Martin Rolim Ribeiro(1), Lilian Osmari Uhlmann ${ }^{(1)}$ e Camila Coelho Becker ${ }^{(1)}$
}

\begin{abstract}
(1)Universidade Federal de Santa Maria,Avenida Roraima, o⒈000, Cidade Universitária, Prédio 77, Sala 2, Bairro Camobi, CEP $97105-900$ Santa Maria, RS, Brasil. E-mail: natalia_schwab@hotmail.com, nstreck2@yahoo.com.br, langnerja@gmail.com, brunasanmartin@hotmail.com, liliuhlmann@yahoo.com.br, camilabecker07@hotmail.com
\end{abstract}

\begin{abstract}
Resumo - O objetivo deste trabalho foi propor um termo para representar o intervalo de tempo de abertura entre flores sucessivas em inflorescências, e verificar a aplicabilidade deste termo a gladíolo de corte. $\mathrm{O}$ termo foi construído pela junção dos radicais gregos anto- $($ antos $=$ flor) e crono- $($ cronos $=$ tempo), para corresponder ao tempo necessário para a abertura de flores sucessivas em ramos florais (inflorescências), tendo-se como unidade o tempo por flor. Para testar o conceito e a aplicabilidade do termo, dados do número acumulado de floretes abertos em espigas de gladíolo foram coletados em dois experimentos de campo, em Santa Maria, RS, de agosto de 2011 a novembro de 2013. Para cada parcela de seis plantas, realizou-se uma regressão linear simples entre o número acumulado de floretes abertos na haste floral e os dias após a emergência das plantas. O termo foi denominado "antocrono" e, em gladíolo, foi estimado como sendo o inverso do coeficiente angular da regressão linear, com a unidade dias por florete. $\mathrm{O}$ antocrono em gladíolo depende da cultivar e decresce com o aumento da temperatura do ar, durante o período de florescimento da espiga.
\end{abstract}

Termos para indexação: Gladiolus x grandiflorus, antese, fenologia, filocrono, gladíolo, inflorescência.

\section{Applicability of the term anthochron for representing the speed rate of flower opening on the inflorescence}

\begin{abstract}
The objective of this work was to propose a term for representing the time interval between the opening of successive flowers on inflorescences and to verify the applicability of this term to gladiola. The term was built by joining the Greek combining forms antho- (anthos $=$ flower) and chron- (chronos = time), to correspond to the time needed for successive flowers to open on floral stems (inflorescences), having time per flower as unit. In order to test the concept and applicability of the term, data on the accumulated number of opened florets on gladiola spikes were collected from two field experiments, in Santa Maria, in the state of Rio Grande do Sul, Brazil, from August 2011 to November 2013. For each plot with six plants, a simple linear regression was performed between the accumulated number of opened florets on the stem and the days after plant emergence. The term was called "anthochron", and in gladiola, it was estimated as the inverse of slope of the linear regression, with the unit days per floret. Anthochron in gladiola depends on the cultivar and decreases with the increase of air temperature during the spike flowering period.
\end{abstract}

Index terms:Gladiolus x grandiflorus, anthesis, phenology, phyllochron, gladiola, inflorescence.

\section{Introdução}

Ao longo dos últimos anos, no Brasil, o setor de floricultura vem experimentando sucessivas taxas de crescimento - da ordem de $8-10 \%$ ao ano em quantidades de produto, e de $12-15 \%$ em valores monetários comercializados -, o que comprova $o$ alto potencial de crescimento da atividade no país (Tombolato et al., 2010). Alguns dos principais produtos comercializados nesse setor são as flores de corte compostas por inflorescência, tais como gladíolo
(Gladiolus x grandiflorus Hort.), boca-de-leão (Antirrhinum majus L.), gipsofila (Gypsophila paniculata L.), esporinha (Consolida ajacis Nieuwl.), tango (Solidago canadenses L.), liatris (Liatris spicata L.) e cúrcuma (Curcuma longa L.). O gladíolo é um importante produto do setor da floricultura (Faraji \& Basaki, 2013; Mushtaq et al., 2013; Ali et al., 2014), e sua parte comercializável é uma haste floral do tipo espiga, que apresenta os botões florais denominados floretes, dispostos sobre um eixo primário (ráquis). 
As inflorescências, como no gladíolo, apresentam desenvolvimento e crescimento afetados pelas condições do ambiente (Shillo \& Halevy, 1976), o que pode incluir alterações da velocidade de abertura das flores que as compõem.

$\mathrm{Na}$ literatura, diversos termos descrevem $\mathrm{o}$ desenvolvimento vegetativo das plantas. Entre eles, destaca-se o termo filocrono, que foi utilizado pela primeira vez por Bunting \& Drennen (1966) e é definido como o intervalo entre o aparecimento de folhas sucessivas em uma haste ou colmo. Atualmente este termo é amplamente utilizado no meio científico e acadêmico para descrever e compreender o desenvolvimento foliar de poáceas (Wilhelm \& McMaster, 1995; Streck et al., 2007; Rosa et al., 2009) e de outras espécies (Dellai et al., 2005; Schuh et al., 2005; Dalmago et al., 2013), inclusive no gladíolo (Streck et al., 2012). Além do filocrono, outros termos utilizados são: plastocrono (Erickson \& Michelini, 1957), primeiramente definido como o intervalo entre a formação de dois entrenós sucessivos e, posteriormente, como o intervalo de tempo entre a iniciação de primórdios sucessivos no ápice meristemático (Esau, 1965); e auxocrono (Hancock \& Barlow, 1960), período entre estádios comparáveis de folhas sucessivas.

No entanto, na literatura, não se encontrou um termo que defina o intervalo de tempo de abertura entre flores sucessivas em inflorescências, ou seja, que constitua a unidade básica do desenvolvimento floral em plantas fanerógamas com inflorescências compostas de flores que abram em sequência. A descrição de um termo que represente a velocidade de abertura das flores, em inflorescências, pode ser útil para calcular a durabilidade ou vida de prateleira das hastes florais, pois poderá basear as práticas de pós-colheita.

O objetivo deste trabalho foi propor um termo para representar o intervalo de tempo entre a abertura de flores sucessivas em inflorescências, e verificar a aplicabilidade deste termo em gladíolo de corte.

\section{Material e Métodos}

Para testar o conceito e a aplicabilidade do termo, foram utilizados dados do número de floretes abertos em espigas de gladíolo (Gladiolus x grandiflorus Hort.), coletados em dois experimentos de campo instalados em uma área de pesquisa, em Santa Maria, RS ( $29^{\circ} 43^{\prime} \mathrm{S}, 53^{\circ} 43^{\prime} \mathrm{W}$, à altitude $\left.95 \mathrm{~m}\right)$. O experimento 1 foi conduzido de agosto de 2011 a novembro de
2012 (12 datas de plantio), e o experimento 2 , de agosto de 2012 a novembro de 2013 (12 datas de plantio), com plantios realizados entre o dia $1^{\circ}$ e o dia 7 de cada mês. Em cada experimento, os tratamentos foram cultivares de gladíolo e épocas de plantio, em delineamento experimental de blocos ao acaso, com arranjo bifatorial e quatro repetições, cada uma delas representada por uma parcela de 10 plantas (total de 40 plantas por tratamento). No experimento 1, foram avaliadas as cultivares de gladíolo 'Peter Pears', 'Rose Friendship' e 'Jester' e, no experimento 2, foram avaliadas 'Amsterdã', 'Rose Friendship' e 'Jester'. Estas cultivares apresentavam diferentes durações do ciclo de desenvolvimento, precoce, médio e tardio, respectivamente. No experimento 1 (2011/2012), as 12 datas de plantio foram 5/8/2011, 2/9/2011, $3 / 10 / 2011,1 / 11 / 2011,1 / 12 / 2011,4 / 1 / 2012,1 / 2 / 2012$, $7 / 3 / 2012,2 / 4 / 2012,2 / 5 / 2012,1 / 6 / 2012$ e $2 / 7 / 2012$; no experimento 2 , (2012/2013) as 12 datas de plantio foram $2 / 8 / 2012,3 / 9 / 2012,4 / 10 / 2012,1 / 11 / 2012$, $3 / 12 / 2012$, 4/1/2013, $1 / 2 / 2013,1 / 3 / 2013,1 / 4 / 2013$, 1/5/2013, 3/6/2013 e 2/7/2013.

Em cada data de plantio, foram utilizados cormos comerciais de gladíolo, com tamanho 14/16 (circunferência externa do cormo, em centímetros), plantados em canteiros de $1 \mathrm{~m}$ de largura por $17 \mathrm{~m}$ de comprimento e $10 \mathrm{~cm}$ de altura, que continham duas linhas espaçadas a $40 \mathrm{~cm}$ e $20 \mathrm{~cm}$ entre plantas na linha. Os blocos foram demarcados nos canteiros e distanciados $1 \mathrm{~m}$ entre si. A adubação de base foi realizada antes do plantio dos cormos no sulco, a $15 \mathrm{~cm}$ de profundidade, na dose de $500 \mathrm{~kg} \mathrm{ha}^{-1}$ da formulação de NPK 05-20-20. Quando as plantas tinham três folhas, realizou-se adubação de cobertura com ureia na dose de $350 \mathrm{~kg} \mathrm{ha}^{-1}$. A irrigação foi aplicada por gotejamento e realizada sempre que necessário, para atender à demanda da cultura, de modo que não ocorresse deficiência hídrica para as plantas. O tutoramento das plantas foi feito com fios de ráfia, presos por estacas de bambu localizadas nas extremidades dos canteiros.

A data de emergência do estande foi definida quando $50 \%$ das plantas de cada bloco apresentavam a brotação visível acima do nível do solo. A partir do primeiro florete aberto, em cada época de plantio, contou-se, duas vezes na semana, o número acumulado de floretes abertos na haste floral, em seis plantas por parcela (repetição), tendo-se deixado uma planta de bordadura de cada lado na linha de plantas, no total de 
24 plantas por cultivar, em cada época. Para o gladíolo, o florete foi considerado aberto quando as anteras estavam visíveis ao observador.

Para cada parcela (repetição), realizou-se uma regressão linear simples entre o número de floretes abertos acumulados na haste floral (NFA) e os dias após a emergência das plantas (DAE). O termo sugerido para representar o tempo necessário para a abertura de flores sucessivas em inflorescências de gladíolo (em dias por florete), foi estimado como sendo o inverso do coeficiente angular da regressão linear, metodologia tradicionalmente usada para estimativa do filocrono (Xue et al., 2004; Koefender et al., 2008; Rosa et al., 2009). A análise estatística foi realizada tendose considerado cada experimento separadamente. As médias da velocidade de abertura de flores em inflorescência de gladíolo foram submetidas à análise de variância, em arranjo bifatorial, para avaliar o efeito das fontes de variação (cultivares e datas de plantio). Nos casos em que a interação cultivares $\mathrm{x}$ datas de plantio foi significativa, a análise foi desdobrada dentro de cada fator. Realizou-se o teste de Tukey, a $5 \%$ de probabilidade, para comparação de médias.

As temperaturas mínima e máxima diárias do ar foram medidas na estação meteorológica automática, do Instituto Nacional de Meteorologia (Inmet), localizada a aproximadamente $150 \mathrm{~m}$ da área experimental.

\section{Resultado e Discussão}

A construção gramatical a partir dos radicais gregos Anto $($ Anto, Antemo $=$ flor $)$ e Crono $($ Crono $=$ tempo $)$ resultou no termo "antocrono". O antocrono indica a velocidade de abertura de flores em inflorescências, já que representa o tempo necessário para a abertura de flores sucessivas em ramos florais (inflorescências). A unidade do antocrono é tempo por flor, e o tempo pode ser expresso em dias. Assim, o antocrono (dias por flor) pode ser facilmente comparado com o filocrono (dias por folha).

A temperatura média do ar à qual as plantas foram expostas, nas diferentes datas de plantio, foi de $7,6^{\circ} \mathrm{C}$ no mês mais frio (julho/2012) até $33,1^{\circ} \mathrm{C}$ no mês mais quente (fevereiro/2012), característica de regiões extratropicais (Figura 1). No experimento 1, o maior valor absoluto de temperatura máxima do ar foi de $39^{\circ} \mathrm{C}$ $(19 / 2 / 2012)$, e o menor valor absoluto de temperatura mínima do ar foi de $-2^{\circ} \mathrm{C}(8 / 6 / 2012)$. No experimento 2 , os valores absolutos de temperatura máxima e de temperatura mínima do ar foram de $39,2^{\circ} \mathrm{C}$, em $1 / 2 / 2013$, e $-0,8^{\circ} \mathrm{C}$ em $25 / 7 / 2013$, respectivamente. A exposição das plantas de gladíolo a diferentes temperaturas do ar é importante para o teste do conceito de antocrono, uma vez que a velocidade de abertura dos floretes pode ser dependente da temperatura do ar, assim como ocorre para o filocrono (Wilhelm \& McMaster, 1995; Streck et al., 2012).

A regressão linear simples entre NFA e DAE, de três cultivares de gladíolo, em duas datas de plantio, para os experimentos 1 e 2, apresentou alto coeficiente de determinação (acima de 0,90), o que indica que a metodologia de estimativa do antocrono (inverso do coeficiente angular de regressão linear) é apropriada, como para o filocrono (Xue et al., 2004; Rosa et al., 2011; Streck et al., 2012) (Figura 2). No plantio de
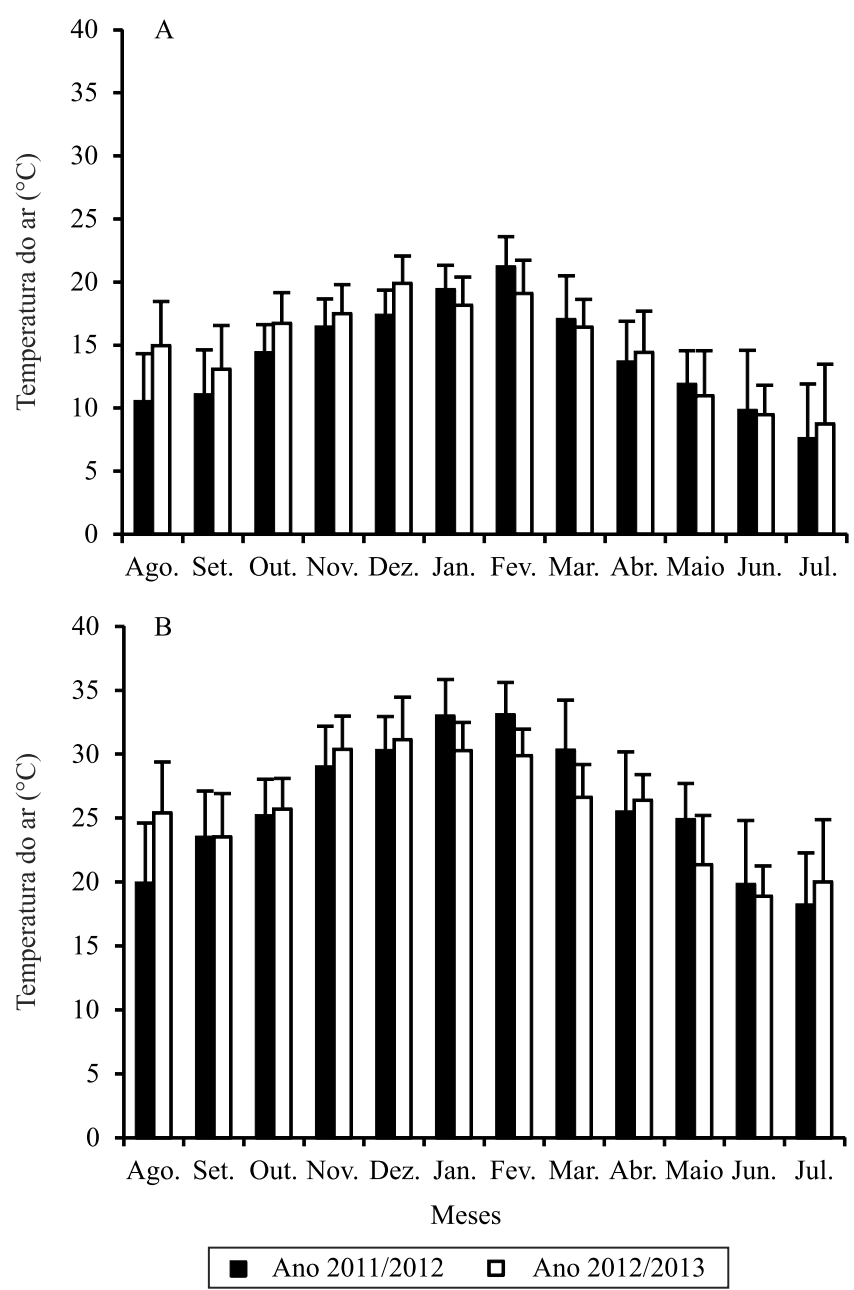

Figura 1. Média mensal de temperatura mínima (A) e máxima (B) do ar, durante dois experimentos, em 2011/2012 e $2012 / 2013$. 

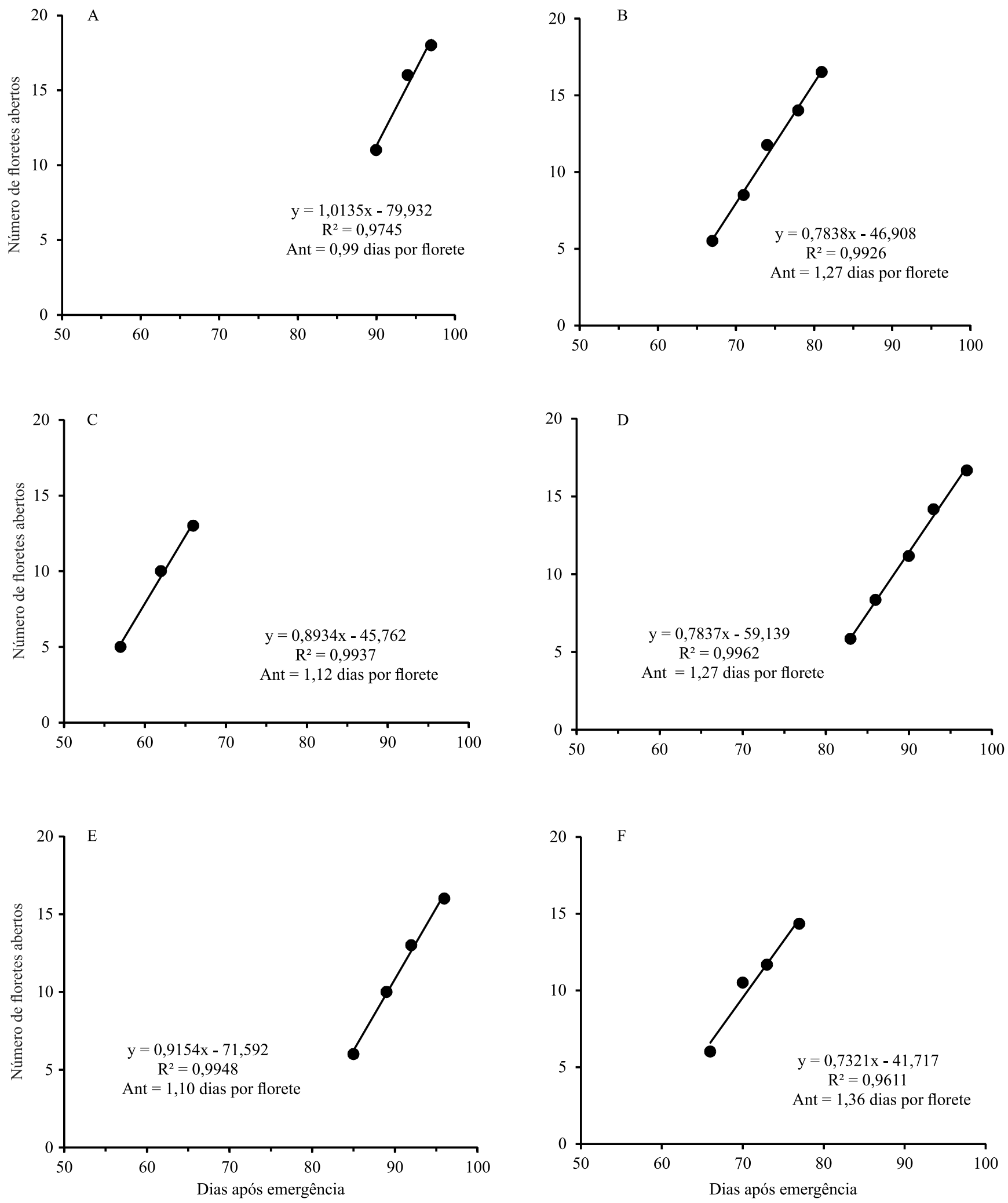

Figura 2. Regressão linear simples entre número de floretes abertos e dias após emergência, no cálculo do antocrono das cultivares de gladíolo 'Amsterdã' (A, B), 'Jester' (C, D) e 'Rose Friendship' (E, F), plantadas em 4/10/2012 (A, C, E) e em $1 / 3 / 2013$ (B, D, F). 
4/10/2012, os floretes abriram na época mais quente do ano (dezembro e janeiro), enquanto no plantio de $1 / 3 / 2013$, os floretes abriram em época mais fria (maio e junho), e o antocrono foi menor na época mais quente (de 0,99 a 1,10 dias por florete) e maior na época fria (1,27 a 1,36 dias por florete). Há também diferenças de antocrono entre as cultivares nas duas épocas. Assim, pressupõe-se que fatores ambientais, como temperatura do ar, umidade do ar e do solo e fotoperíodo, e fatores genéticos, como espécie e cultivar, podem afetar o antocrono, assim como já evidenciado para o filocrono (Wilhelm \& McMaster, 1995; El-Shafi \& El-Mohsen, 2013).

Houve efeito significativo da interação cultivares $\mathrm{x}$ datas de plantio sobre o antocrono nos dois experimentos (Tabela 1). O efeito de data de plantio é evidente em

Tabela 1. Média do antocrono de quatro cultivares de gladíolo, ao longo de diversas datas de plantio, em dois anos de cultivo ${ }^{(1)}$.

\begin{tabular}{|c|c|c|c|}
\hline \multirow[t]{2}{*}{ Data } & \multicolumn{3}{|c|}{ Antocrono (dia por florete) } \\
\hline & $\begin{array}{l}\text { 'Peter Pears' ou } \\
\text { 'Amsterdã'(2) }\end{array}$ & 'Jester' & $\begin{array}{c}\text { 'Rose } \\
\text { Friendship' }\end{array}$ \\
\hline $5 / 8 / 2011$ & $0,65 \mathrm{Cb}$ & $0,95 \mathrm{BCa}$ & - \\
\hline 2/9/2011 & $0,86 \mathrm{BCa}$ & $0,71 \mathrm{Cb}$ & $0,97 \mathrm{Aba}$ \\
\hline $3 / 10 / 2011$ & $0,83 \mathrm{BCa}$ & $0,71 \mathrm{Cb}$ & 0,81Aba \\
\hline $1 / 11 / 2011$ & $0,87 \mathrm{BCb}$ & $1,07 \mathrm{BCab}$ & $1,26 \mathrm{Aa}$ \\
\hline $1 / 12 / 2011$ & $0,72 \mathrm{Ca}$ & $0,82 \mathrm{BCa}$ & $0,69 \mathrm{Ba}$ \\
\hline $4 / 1 / 2012$ & $0,82 \mathrm{BCa}$ & $0,81 \mathrm{BCa}$ & $1,04 \mathrm{Aba}$ \\
\hline $1 / 2 / 2012$ & 1,51Aa & $1,03 \mathrm{BCb}$ & - \\
\hline $7 / 3 / 2012$ & - & 1,93 Aa & $0,89 \mathrm{ABb}$ \\
\hline $2 / 4 / 2012$ & - & - & - \\
\hline $2 / 5 / 2012$ & - & $1,21 \mathrm{Ba}$ & 1,01Aba \\
\hline $1 / 6 / 2012$ & $1,08 \mathrm{Ba}$ & $0,88 \mathrm{BCa}$ & $1,30 \mathrm{Aa}$ \\
\hline 2/7/2012 & - & $1,14 \mathrm{BCa}$ & $1,25 \mathrm{Aa}$ \\
\hline CV (\%) & \multicolumn{3}{|c|}{17,67} \\
\hline 2/8/2012 & $0,77 \mathrm{DEa}$ & $0,75 \mathrm{Ca}$ & $0,87 \mathrm{Aa}$ \\
\hline $3 / 9 / 2012$ & $0,64 \mathrm{Ea}$ & $0,71 \mathrm{Ca}$ & $0,82 \mathrm{Aa}$ \\
\hline 4/10/2012 & $0,88 \mathrm{CDEa}$ & $1,36 \mathrm{Ba}$ & 1,09Aa \\
\hline $1 / 11 / 2012$ & $0,81 \mathrm{DEb}$ & $2,07 \mathrm{Aa}$ & - \\
\hline $3 / 12 / 2012$ & $1,15 \mathrm{ABCDa}$ & $1,13 \mathrm{BCa}$ & - \\
\hline $4 / 1 / 2013$ & $1,326 \mathrm{Aba}$ & $0,93 \mathrm{BCa}$ & $1,18 \mathrm{Aa}$ \\
\hline $1 / 2 / 2013$ & 0,92BCDEa & $0,87 \mathrm{BCa}$ & $0,78 \mathrm{Aa}$ \\
\hline $1 / 3 / 2013$ & $1,28 \mathrm{ABCa}$ & $1,28 \mathrm{BCa}$ & $1,33 \mathrm{Aa}$ \\
\hline $1 / 4 / 2013$ & - & $0,91 \mathrm{BCa}$ & $1,43 \mathrm{Aa}$ \\
\hline $1 / 5 / 2013$ & - & $1,35 \mathrm{Ba}$ & $1,49 \mathrm{Aa}$ \\
\hline $3 / 6 / 2013$ & $1,39 \mathrm{Aa}$ & $1,06 \mathrm{BCab}$ & $0,89 \mathrm{Ab}$ \\
\hline 2/7/2013 & 0,89CDEa & $0,91 \mathrm{BCa}$ & $1,02 \mathrm{Aa}$ \\
\hline CV (\%) & \multicolumn{3}{|c|}{19,68} \\
\hline
\end{tabular}

(1)Médias seguidas de letras iguais, maiúsculas nas colunas e minúsculas nas linhas, não diferem entre si, pelo teste de Tukey, a $5 \%$ de probabilidade. (2)'Peter Pears' de 5/8/2011 a 2/7/2012 e 'Amsterdã' de 2/8/2012 a 2/7/2013. todas as cultivares, exceto para a 'Rose Friendship', no experimento 2 (ano 2012/2013), que não apresentou diferença estatística quanto ao antocrono nas diferentes datas. Foram encontradas diferenças estatísticas entre as cultivares em oito datas de plantio $(5 / 8 / 11,2 / 9 / 11$, $3 / 10 / 11,1 / 11 / 11,1 / 2 / 12,7 / 3 / 12,1 / 11 / 12$ e $3 / 6 / 13)$, e nas outras 16 datas não houve diferença de antocrono entre as cultivares, o que indica que a variação de antocrono resulta mais das datas de plantio do que das cultivares. Em trigo, estudos realizados por El-Shafi \& El-Mohsen (2013) apontaram maior efeito genotípico do que da temperatura do ar sobre o filocrono. Streck et al. (2007) estudaram arroz irrigado e afirmaram que o ambiente em que as plantas se desenvolvem afeta mais o filocrono do que o genótipo, o que está de acordo com os resultados encontrados para gladíolo no presente trabalho.

Um fator que pode explicar o efeito da data de plantio sobre o antocrono (Tabela 1) é a temperatura do ar, já que, para as quatro cultivares de gladíolo, testadas em dois experimentos, ao longo de 12 datas de plantio, o antocrono decresceu com o aumento da temperatura do ar durante o período de abertura dos floretes (Figura 3). Esta relação negativa do antocrono com a temperatura do ar foi estatisticamente significativa para as quatro cultivares de gladíolo (Figura 4 A, B, C e D). A relação da velocidade de abertura dos floretes

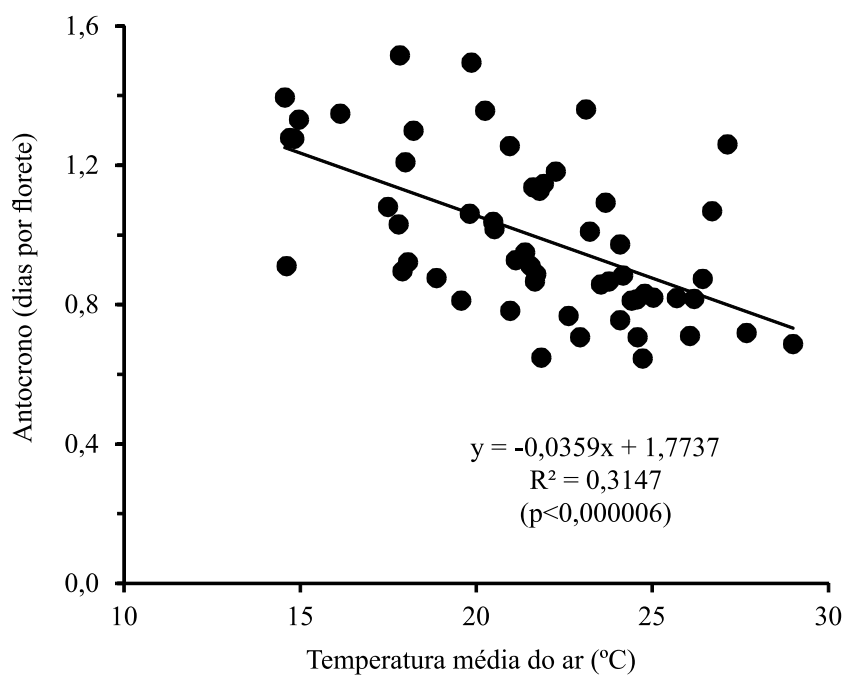

Figura 3. Antocrono de gladíolo em função da temperatura média do ar, durante o período de floração da espiga. Dados das cultivares de gladíolo 'Peter Pears', 'Amsterdã', 'Jester' e 'Rose Friendship', plantadas ao longo de 12 épocas em dois anos de cultivo (agosto/2011 a julho/2012 e agosto/2012 a julho/2013). 
com a temperatura do ar é fisiologicamente similar à dependência da velocidade de emissão de folhas com a temperatura do ar (Xue et al, 2004; Streck et al., 2009b, 2012), o que indica a oportunidade de se utilizar a soma térmica como unidade de tempo no conceito do antocrono em estudos futuros. No entanto, para calcular a soma térmica necessita-se conhecer a temperatura basal inferior $(\mathrm{Tb})$ do processo biológico e, no caso da abertura de floretes em gladíolo, a $\mathrm{Tb}$ ainda não é conhecida.

Do ponto de vista comercial, a relação entre a velocidade de abertura de floretes do gladíolo e temperatura do ar tem importância para a pós-colheita (Figura 3 e 4), o que indica que hastes florais mantidas a altas temperaturas $\left(25\right.$ a $\left.30^{\circ} \mathrm{C}\right)$ podem ter vida de prateleira menor do que as mantidas a temperaturas mais amenas $\left(15 \mathrm{a} 20^{\circ} \mathrm{C}\right)$. Silva et al. (2008) afirmam que quanto menor a temperatura de armazenamento para hastes florais de gladíolo, maior a durabilidade das flores para o consumidor; porém, Nowak et al. (1992) salientam que flores de origem tropical não devem ser armazenadas a temperaturas fora da faixa que varia de 7 a $15^{\circ} \mathrm{C}$, em razão da maior probabilidade de ocorrência de danos por frio (denominados “chilling”),
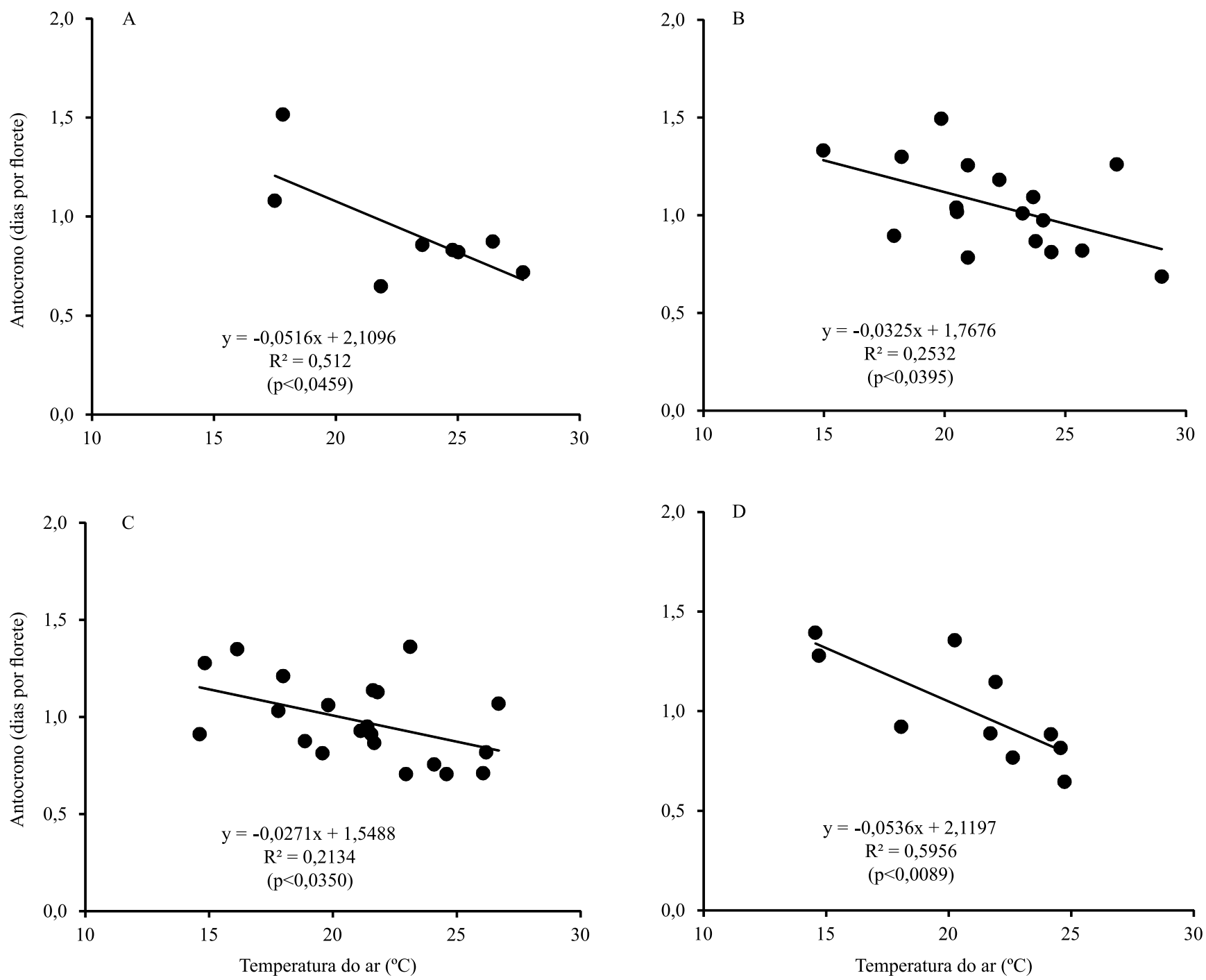

Figura 4. Antocrono de gladíolo em função da temperatura média do ar, durante o período de floração da espiga, das cultivares (A) 'Peter Pears', (B) 'Rose Friendship', (C) 'Jester' e (D) 'Amsterdã'. Dados de 12 épocas de plantio, em dois anos de cultivo (agosto/2011 a julho/2012 e agosto/2012 a julho/2013). 
que apresentam como sintomas a descoloração e lesões necróticas das pétalas, além do atraso da abertura dos botões após o armazenamento.

No presente trabalho, o termo antocrono foi sugerido para representar a velocidade de abertura de flores em inflorescências. $O$ termo pode ser aplicado principalmente a espécies que apresentam inflorescências classificadas botanicamente como "indefinidas", em que a abertura das flores se dá no sentido acropetal (de baixo para cima), tais como as do tipo rácimo ou cacho (ex: Vitis vinifera L., Crotalaria zanzibarica Benth. e Consolida ajacis Nieuwl.), espiga (ex: Gladiolus x grandiflorus Hort., Liatris spicata L. e Curcuma longa L.), panícula (ex: Oryza sativa L. e Gypsophila paniculata L.) e espádice (ex: Monstera deliciosa Liebm.).

Assim, a aplicabilidade do termo antocrono, a exemplo do conceito de filocrono (Wilhelm \& McMaster, 1995), pode ser estendida a outras espécies vegetais além do gladíolo, como o arroz e o trigo. Em arroz, a determinação do antocrono se iniciaria no estágio R4 da escala de Counce et al. (2000). Em trigo, a abertura das flores se inicia na porção central da espiga e se estende para a base e para o ápice (Zadoks et al., 1974) e, neste caso, o antocrono também pode ser determinado pelo número de flores abertas, tanto para baixo quando para cima, a partir do centro da espiga.

A determinação do antocrono pode ser realizada por análise de regressão entre o número de flores abertas na inflorescência e o tempo (dias) após a emergência da cultura (Figura 2). Essa é a metodologia canônica de estimativa do filocrono, realizada por regressão linear entre o número de folhas acumuladas em uma haste e o tempo (em dias ou ${ }^{\circ} \mathrm{C}$ dia) após a emergência da cultura (Wilhelm \& McMaster, 1995; Streck et al., 2012).

Outra aplicação do termo antocrono é na determinação da duração do período de florescimento, que ocorre entre a abertura da primeira e da última flor da inflorescência. Para o filocrono, a duração do período de desenvolvimento foliar - emergência da cultura até o aparecimento da folha bandeira - é uma função da velocidade do aparecimento de cada folha (filocrono) e do número final de folhas na haste principal (Streck et al., 2007, 2009a, 2012). Assim, para o antocrono, a duração do período de florescimento é uma função da velocidade de abertura das flores (antocrono) e do número final de flores na inflorescência. Para o gladíolo, o período de florescimento corresponde ao tempo máximo (dias) de durabilidade das hastes florais.

\section{Conclusões}

1. O termo antocrono é aplicável para definir o intervalo de tempo entre a abertura de flores sucessivas em inflorescências, com unidade tempo por flor.

2. Em gladíolo de corte, o antocrono (em dias por florete) depende da cultivar e decresce com o aumento da temperatura do ar durante o período de floração da espiga.

\section{Agradecimentos}

À Coordenação de Aperfeiçoamento de Pessoal de Nível Superior (Capes) e ao Conselho Nacional de Desenvolvimento Científico e Tecnológico (CNPq), pelas bolsas de estudo e de produtividade.

\section{Referências}

ALI, A.; MEHMOOD, T.; HUSSAIN, R.; BASHIR, A.; NAJAM-UD-DIN, S.; AHMAD, A. Investigation of biofertilizers influence on vegetative growth, flower quality, bulb yield and nutrient uptake in gladiolus (Gladiolus grandiflorus L.). International Journal of Plant, Animal and Environmental Sciences, v.4, p.94-99, 2014.

BUNTING, A.H.; DRENNEN, D.S.H. Some aspects of the morphology and physiology of cereal in the vegetative phase. In: EASTER SCHOOL IN AGRICULTURE SCIENCE, 12., 1966, London. The growth of cereal and grasses: proceedings. London: University of Nottingham, 1966. p.20-38.

COUNCE, P.A.; KEISLING, T.C.; MITCHELL, A.J. A uniform, objective, and adaptive system for expressing rice development. Crop Science, v.40, p.436-443, 2000. DOI: 10.2135/ cropsci2000.402436x.

DALMAGO, G.A.; FOCHESATTO, E.; KOVALESKI, S.; TAZZO, I.F.; BOLIS, L.M.; CUNHA, G.R. da; NIED, A.H.; BERGAMASCHI, H.; SANTI, A. Filocrono e número de folhas da canola em diferentes condições ambientais. Pesquisa Agropecuária Brasileira, v.48, p.573-581, 2013. DOI: 10.1590/ S0100-204X2013000600001.

DELlAI, J.; TRENTIN, G.; BISOGNIN, D.A.; STRECK, N.A. Filocrono em diferentes densidades de plantas de batata. Ciência Rural, v.35, p.1269-1274, 2005. DOI: 10.1590/ S0103-84782005000600007.

EL-SHAFI, M.A.A.; EL-MOHSEN, A.A.A. Predicting the effect of temperature on leaf appearance in seven spring bread wheat genotypes. World Essays Journal, v.1, p.46-51, 2013.

ERICKSON, R.O.; MICHELINI, F.J. The plastochron index. American Journal of Botany, v.44, p.297-305, 1957. DOI: $10.2307 / 2438380$.

ESAU, K. Plant anatomy. New York: John Wiley \& Sons, 1965. $767 \mathrm{p}$. 
FARAJI, S.; BASAKI, T. Evaluation of plant growth regulators on phonologic stages and morphologic traits of gladiolus (White prosperity cultivar). International Journal of Agronomy and Plant Production, v.4, p.1549-1551, 2013.

HANCOCK, C.R.; BARLOW, H.W.B. Short-term fluctuations in leaf and inter-node growth in the apple root stock Crab C. New Phytologist, v.59, p.227-237, 1960. DOI: 10.1111/j.14698137.1960.tb06218.x.

KOEFENDER, J.; STRECK, N.A.; BURIOL, G.A.; TRENTIN, R. Estimativa do filocrono em calêndula. Ciência Rural, v.38, p.1246-1250, 2008. DOI: 10.1590/S0103-84782008000500007.

MUSHTAQ, S.; HAFIZ, I.A.; IQBAL, M.S.; HASAN, S.Z. ul; ARIF, M.; ULLAH, S.; RASHEED, M.; RAFIQUE, R. Studies on the performance of some exotic gladiolus cultivars under rain-fed conditions. International Journal of Modern Agriculture, v.2, p.108-113, 2013.

NOWAK, J.; RUDNICKI, R.M.; DUNCAN, A.A. Postharvest handling and storage of cut flowers, florist greens, and potted plants. Portoland: Timber Press, 1992. 210p.

ROSA, H.T.; WALTER, L.C.; STRECK, N.A.; ALBERTO, C.M. Métodos de soma térmica e datas de semeadura na determinação de filocrono de cultivares de trigo. Pesquisa Agropecuária Brasileira, v.44, p.1374-1382, 2009. DOI: 10.1590/ S0100-204X2009001100002.

ROSA, H.T.; WALTER, L.C.; STRECK, N.A.; ANDRIOLO, J.L.; SILVA, M.R. da; LANGNER, J.A. Base temperature for leaf appearance and phyllochron of selected strawberry cultivars in a subtropical environment. Bragantia, v.70, p.939-945, 2011. DOI: 10.1590/S0006-87052011000400029.

SCHUH, M.; STRECK, N.A.; NARDI, C.; BURIOL, G.A.; BELLÉ, R.A.; BRACKMANN, A. Vernalização afeta o filocrono em lírio. Bragantia, v.64, p.25-32, 2005. DOI: 10.1590/ S0006-87052005000100003.

SHILLO, R.; HALEVY, A.H. Inflorescence development of flowering and blasted gladiolus plants in relation to development of other plant parts. Scientia Horticulturae, v.4, p.79-86, 1976. DOI: 10.1016/0304-4238(76)90068-6.
SILVA, L.R. da; OLIVEIRA, M.D. de M.; SILVA, S. de M. Manejo pós-colheita de hastes florais de gladíolos (Gladiolus grandiflorus L.). Acta Agronómica, v.57, p.129-135, 2008.

STRECK, N.A.; BELLÉ, R.A.; BACKES, F.A.A.L.; GABRIEL, L.F.; UHLMANN, L.O.; BECKER, C.C. Desenvolvimento vegetativo e reprodutivo em gladíolo. Ciência Rural, v.42, p.1968-1974, 2012. DOI: 10.1590/ S0103-84782012001100010.

STRECK, N.A.; DE PAULA, F.L.M.; DELLAI, J.; BISOGNIN, D.A.; DE PAULA, A.L. Filocrono em batateira afetado pelo tamanho do tubérculo-semente e pela época de cultivo. Bragantia, v.68, p.137-143, 2009a. DOI: 10.1590/ S0006-87052009000100015.

STRECK, N.A.; LAGO, I.; SAMBORANHA, F.K.; GABRIEL, L.F.; SCHWANTES, A.P.; SCHONS, A. Temperatura base para aparecimento de folhas e filocrono da variedade de milho BRS Missões. Ciência Rural, v.39, p.224-227, 2009b. DOI: 10.1590/S0103-84782009000100035.

STRECK, N.A.; MICHELON, S.; ROSA, H.T.: WALTER, L.C.; BOSCO,L.C.; DEPAULA, G.M.; CAMERA, C.; SAMBORANHA, F.K.; MARCOLIN, E.; LOPES, S.J. Filocrono de genótipos de arroz irrigado em função de época de semeadura. Ciência Rural, v.37, p.323-329, 2007. DOI: 10.1590/S0103-84782007000200005.

TOMBOLATO, A.F.C.; UZZO, R.P.; JUNQUEIRA, A.H.; PEETZ, M. da S.; STANCATO, G.C.; ALEXANDRE, M.A.V. Bulbosas ornamentais no Brasil. Revista Brasileira de Horticultura Ornamental, v.16, p.127-138, 2010.

WILHELM, W.; MCMASTER, G.S. Importance of the phyllochron in studying development and growth in grasses. Crop Science, v.35, p.1-3, 1995. DOI: 10.2135/cropsci1995.0011183X0035000 $10001 \mathrm{x}$.

XUE, Q.W.; WEISS, A.; BAENZIGER, P.S. Predicting leaf appearance in field-grown winter wheat: evaluating linear and non-linear models. Ecological Modelling, v.175, p.261-270, 2004. DOI: $10.1016 /$ j.ecolmodel.2003.10.018.

ZADOKS J.C.; CHANG, T.T.; KONZAK, C.F. A decimal code for the growth stages of cereals. Weed Research, v.14, p.415-421, 1974. DOI: 10.1111/j.1365-3180.1974.tb01084.x. 